\title{
More precise solar-limb light-bending
}

A gargantuan exercise in data-analysis has produced the most accurate value yet of the deflection of radiation travelling near the limb of the Sun.

Historically, the most dramatic proof that Einstein was on the right track with his general theory of relativity came in 1919, when Eddington and his associate Crommelin took themselves off to the western Pacific and the track of the total eclipse of the Sun in that year. They were bent on measuring the predicted deflection of light from distant stars lying near the limb of the Sun. As the world knows, the prediction was confirmed within the limits of the accuracy of the observations, to general applause. Einstein became a celebrity overnight.

What is not so well remembered is that the measurements in 1919 were not particularly accurate. The standard error of the measurements was roughly 30 per cent, while the displacement of the images of different stars in the field near the eclipsed Sun ranged from a half to twice the deflection Einstein had predicted. And later eclipse measurements have not been much better, largely because of the difficulties of making accurate measurements from the ad hoc observatories that have to be established.

Luckily, it is no longer necessary to wait for successive solar eclipses to improve on the Eddington result. More than 30 years ago, Irwin Shapiro (then and still at MIT) argued that the then-recently discovered radio-quasars would be more convenient and potentially more accurate ways of measuring the deflection of radiation near the limb of the Sun. If they happen to be in the sky, they have the advantage of being observable at all times, while those that happen to be in or near the Ecliptic will regularly come into near occultation of the Sun.

Shapiro's case is now well established. Since 1968, when the first radio results were published, the error of the measured deflection near the limb of the Sun has been steadily reduced, from something like 10 per cent to much less than 1 per cent. And, satisfactorily as it must seem, increasing accuracy has seen the estimated value of the deflection converge on Einstein's prediction. Is that not a sign that the further refinement of this test of general relativity can be safely put on the back burner?

Not so, and for good reasons, as will be apparent. The latest development is the use of a VLBI (for "Very Long Baseline Interferometry") technique for a direct measurement of the time delay in signals from radio-quasars passing near the limb of the Sun. Shapiro appears to have been the first to point out in 1967 that the deflection of a light-beam would be accompanied by a delay, and that the two quantities are simply related to each other; it is pleasing that he is one of the authors of the new measurement, whose uncertainty appears to be 0.2 per cent (D. E. Lebach $e t$ al.Phys. Rev. Lett. 75, 1439-1442; 21 August 1995). Those concerned are from the Harvard-Smithsonian Center for Astrophysics, the Haystack Observatory and from MIT.

The long baseline on which the measurement is based spans the distance between the Haystack Observatory and the Owens Valley Observatory in California. The target radio-source was the quasar 3C279; observations were made during an eight-day period on either side of the occultation of that source by the Sun in October 1987 (near the most recent sunspot minimum). To control for certain sources of systematic error, a nearby radiosource, $3 \mathrm{C} 273 \mathrm{~B}$, separated by $10^{\circ}$ on the sky, was used as a standard. Because of the need to observe at three different frequencies, two different radiotelescopes were used at each site. All four had to be switched between the target source and the control in synchrony, in practice on a 8minute cycle.

The complexity of the data reduction evidently accounts for some of the delay in the appearance of this important result. (Another explanation is that Lebach's PhD thesis is cited as a source of further information.) Apart from the expected phase delay caused by the gravitational field of the Sun (not to mention that of the Earth), the effects of the Earth's atmospshere and interstellar plasma have to be allowed for. The three different frequency bands were, in reality, subdivided into fourteen, within each of which the signals received at the two sites were cross-correlated to yield estimates of the phase delay. It turned out to be necessary to allow for discrepancies between the atomic clocks at the two ends of the baseline, which is possible only by internal analysis of the data. Interestingly, one of the chief contributions to the eventual error of the measurement is the uncertainty about the relationship of the two sets of radiotelescopes to the Earth's axis, which inevitably affects the group's choice of a reference frame.

The conventional way of stating the outcome of both the time-delay and deflection measurements is in terms of a quantity which turns up in the predictions as $(1+\gamma)$, where $\gamma$ is a quantity calculated from the general theory of relativity to be exactly unity. Thus the deflection of a light-ray near the limb of the Sun turns out to be proportional to $(1+\gamma) / \delta$, where $\delta$ is the distance between the ray and the surface of the Sun as a fraction of the solar radius. The time-delay is similarly proportional to $(1+\gamma)$.

In each case, Einstein's relativity is verified if $\gamma=1$ within the limits of error. Lebach et al. report that $\gamma=0.9996 \pm$ 0.0017 , which is as much as anybody could ask for at this stage. But two of the authors, Shapiro and J. L. Davis, say that they now plan to work through the VLBI data collected in recent decades for geodetic purposes; they hope they can thus improve the precision of their data several-fold. (It is relevant that sensitive measurements of extra-galactic radio sources already make allowance for the Sun's gravity, even when the objects are a long way away from the Sun in the sky.)

What does any of this matter? There is more than academic interest in the search for a more precise $\gamma$. If, for example, should somebody seek to modify the general theory, he or she should ensure that the timedelay (or deflection) of light-rays near the limb of the Sun is properly accounted for. After all, the Brans-Dicke modification (invented in the 1960 s by adding a scalar field to the ten-component tensor field of Einstein's space-time metric) was in part discarded on the strength of early radioquasar measurements.

Yet these are still relatively local measurements. Moreover, the prediction of light-bending follows directly from Einstein's general theory in, so to speak, local mode, and does not depend on the choice of a particular solution of his equations to represent the Universe as a whole. If eventually modifications of general relativity are found necessary, they will surely be in be applicable only where the gravitational field is much stronger than in the neighbourhood of the boring old Sun (which nobody has yet thought it worthwhile to designate as a black hole, although that may not take long the way things are going).

The crying need remains what it has been for the past two decades, that of marrying together general relativity and quantum mechanics. As things are, they are like chalk and cheese. It will be a great surprise if general relativity survives that marriage unchanged.

John Maddox 